\title{
Vision-targeted health related quality of life crossuat in older adults: patient-reported visibility problems in low luminance activities are more likely to decline than daytime activities
}

Cynthia Owsley ${ }^{1 *}$ (D) and Gerald McGwin Jr ${ }^{1,2}$

\begin{abstract}
Background: Commonly used vision-targeted health-related quality of life questionnaires almost exclusively focus items on vision under daytime conditions. Older adults even when in good eye health frequently report experiencing vision problems at night and under low environmental light levels, and psychophysical studies also document these visibility problems. Here we compare the progression of self-reported low luminance visibility problems and self-reported visibility problems under daytime conditions in older adults.

Methods: Trained interviewers administered two questionnaires to older adults in normal eye health: the National Eye Institute Visual Function Questionnaire - 25 (NEI VFQ-25) where items are almost entirely focused on difficulties in daytime activities, and the Low Luminance Questionnaire (LLQ) where items are focused on difficulties seeing at night and under low luminance conditions. The following visual functions were also measured: visual acuity, low luminance visual acuity, low luminance deficit, contrast sensitivity, light sensitivity in the macula, and rod-mediated dark adaptation. The protocol was repeated 3 years later.

Results: Scores on the NEI VFQ-25 composite and its subscales were unchanged between baseline and 3-year follow-up, whereas scores on the LLQ composite and 5 of 6 subscales significantly decreased (corresponding to less functionality) at the 3-year follow-up. Participants were more likely to display a $\geq 5$ point decrease on the LLQ composite than on the NEI VFQ-25 over 3 years. Visual functional tests were largely unrelated to changes in NEI VFQ-25 and LLQ scores from baseline to follow-up.

Conclusions: Older adults' vision-targeted quality of life as measured by questionnaire is more likely to exhibit a practically significant decrease over 3 years using a questionnaire that focused on low luminance activities (LLQ) than one focused on daytime activities (NEI VFQ-25). That the results of visual functional testing did not correspond to older adults' decline in self-reported problems in low luminance activities emphasizes the importance of questionnaires in understanding visual difficulties from the patients' own perspective.
\end{abstract}

Keywords: Aging, Vision, Quality of life, Questionnaire, Visual activities, Low luminance, Night vision

\footnotetext{
*Correspondence: owsley@uab.edu

${ }^{1}$ Department of Ophthalmology, School of Medicine, University of Alabama

at Birmingham, Birmingham, AL 35294-0009, USA

Full list of author information is available at the end of the article
} 


\section{Background}

Older adults experience aging-related challenges in the visual activities of daily living, even in the absence of the common eye conditions and diseases of later adulthood (e.g., cataract, age-related macular degeneration (AMD), glaucoma, diabetic retinopathy). They frequently report experiencing vision problems at night and under low environmental light levels. For example, they cite difficulty with night driving and often avoid it [1-3] and report task difficulties under low illumination (e.g., reading a menu in a dimly lit restaurant) [4]. Psychophysical studies confirm that older adults, even when free of significant ocular conditions, tend to exhibit decreased scotopic and mesopic light sensitivity, contrast sensitivity, and acuity, as compared to younger adults [5-10].

It is thus concerning that commonly used visiontargeted health-related quality of life questionnaire instruments designed for use in the older adult population almost exclusively focus items on vision under photopic (day-time) conditions, such as the National Eye Institute Visual Function Questionnaire (NEI VFQ-25,) [11] among others $[12,13]$. Thus, these questionnaire instruments do not adequately address one of the major vision problem areas cited by older adults, activities at lower ambient light levels. In response to a need for a questionnaire focused on low luminance content, a questionnaire was recently developed specifically targeted at this content; the Low Luminance Questionnaire (LLQ) is a 32-item questionnaire designed for use with older adults and has established content and construct validity and test-retest reliability $[14,15]$ The LLQ is accessible at http://www.uab.edu/ medicine/ophthalmology/images/Research/Low\%20Lu minance.pdf). It has six subscales: driving, extreme lighting, mobility, emotional distress, general dim lighting, peripheral vision.

Even though considerable research has shown that older adults' vision problems are exacerbated under low luminance as measured psychophysically and by selfreport, it remains to be determined whether self-reported low luminance visibility problems progress more rapidly over time in older adults as they age as compared to visibility issues they cite under daytime conditions. Here we examine whether responses on the LLQ display larger decreases over 3 years in older adults free of significant ocular conditions, as compared to responses on the NEI VFQ-25, which is largely focused on daytime activities. We also examined whether decreases in questionnaire scores were related to visual function at baseline and change in visual function over a 3-year period of follow-up.

\section{Method}

The protocol (\#F080205001) was approved by the Institutional Review Board of the University of Alabama at
Birmingham (UAB) and followed the tenants of the Declaration of Helsinki. Informed consent was obtained from all participants after the nature and purpose of the study was described. This study made use of the sample assembled for the Alabama Study on Early Age-Related Macular Degeneration (ALSTAR), a prospective study of older adults in normal macular health at baseline $[16,17]$. As described previously, participants were recruited from two primary care ophthalmology practices in the Callahan Eye Hospital at UAB. Eligibility criteria were as follows: (1) age $\geq 60$ years old; (2) normal macular health in both eyes as determined by 3 -field digital stereo-fundus photos (Carl Zeiss Meditec 450 Plus camera, Dublin, CA) evaluated by an experienced grader masked to other study variables. Each eye's grade had to be 1 in the Age-Related Eye Disease Study (AREDS) 9-step classification system [18], indicating normal macular health. (3) No previous diagnoses of glaucoma, other retinal conditions, optic nerve conditions, corneal disease, diabetes, Alzheimer's disease, Parkinson's disease, brain injury, other neurological or psychiatric conditions as revealed by the medical record or by self-report.

A baseline visit consisted of the following. Participants provided information on demographic characteristics (age, gender, race/ethnicity). The NEI VFQ-25 and the LLQ were interviewer administered. Both of these instruments were developed using classical test theory, [19] and we elected to score each instrument using recommended scoring instructions by their developers $[11,14]$ The NEI VFQ-25 and the LLQ composite and subscale scores are computed using the same method, that is, by scaling individual item responses from 0 to 100 , where 100 represents the highest functional level and 0 the lowest, and then averaging the individual items. Other options for scoring these types of questionnaires include a collection of measurement models referred to as item response theory [19]. General cognitive status was estimated by the MiniMental State Examination (MMSE) [20]; scores less than 24 indicated cognitive impairment.

Several visual function tests were also administered; tests were administered for each eye unless otherwise noted. The eye with better visual function was used in analyses. Best-corrected visual acuity for each eye was assessed via the Electronic Visual Acuity tester [21] (EVA; JAEB Center, Tampa FL) under photopic conditions $\left(100 \mathrm{~cd} / \mathrm{m}^{2}\right)$ and expressed as the logarithm of the minimum angle resolvable (logMAR). The EVA was also used to assess low luminance visual acuity for each eye, with participants viewing letters through a $1.5 \mathrm{log}$ unit neutral density filter [22]. The filter reduced background luminance to $3.1 \mathrm{~cd} / \mathrm{m}^{2}$. To determine how much $\log$ MAR decreased under conditions of the lower light level as compared to the photopic $\left(100 \mathrm{~cd} / \mathrm{m}^{2}\right)$ assessment, we defined a decrease in visual acuity under low luminance 
by the increase in $\log$ MAR (referred to as the "low luminance deficit" [22]). Contrast sensitivity for each eye was estimated by the Pelli-Robson chart [23] (Precision Vision, La Salle, IL) with mean luminance of $100 \mathrm{~cd} / \mathrm{m}^{2}$, the letter-by-letter scoring method [24], and expressed as logarithm of sensitivity. Macular light sensitivity for each eye was assessed using the Humphrey Field Analyzer (Carl Zeiss Meditec, Dublin, CA) and the 24-2 SITA standard protocol, as previously described [25]. Sensitivities for 16 test targets within a $9^{\circ} \times 9^{\circ}$ macular region were averaged, and expressed as decibels (dB). Rod-mediated dark adaptation was measured using a computerized dark adaptometer as described previously $[16,17]$. Test targets with a diameter of $2^{\circ}$ were positioned at $5^{\circ}$ on the inferior vertical meridian (superior to the fovea on the retina). Following a photobleach exposure (equivalent $\sim 83 \%$ bleach), sensitivity was measured at $30-\mathrm{s}$ intervals for $20 \mathrm{~min}$ following bleach offset. Dark adaptation time was defined by the rod intercept, the time in minutes needed by the participant to reach a criterion sensitivity value in the latter half of the second component of rod recovery. Due to time constraints in the protocol visit, dark adaptation was measured in one eye only.

Administration of the NEI VFQ-25, LLQ, and visual function testing was repeated 3 years after the baseline visit. The only exception was measurement of macular light sensitivity, which was not repeated at the 3-year follow-up due to time constraints in the study protocol. The eye with better visual function was used in analyses.

Baseline and 3-year follow-up NEI-VFQ and LLQ composite and subscale scores were compared using paired t-tests. Unpaired t-tests were used to compare participants whose LLQ composite did and did not decrease by five or more points with respect to visual function variables. A similar analysis was conducted for the NEI-VFQ composite. P-values less than 0.05 (twotailed) were considered statistically significant.

\section{Results}

There were 365 participants in the ALSTAR study in normal eye health who completed both the baseline and follow-up LLQ and NEI VFQ-25. The vast majority of the sample were in their 60 s or 70 s $(97.5 \%)$ and were white of non-Hispanic origin (94.5\%) (Table 1). Approximately $2 / 3$ of the sample was women. In terms of cognitive status, $98.1 \%$ (358 of 365) had MMSE scores in the non-cognitively impaired range (24-30).

Table 2 shows the mean and standard deviation for the NEI VFQ-25 composite and subscale scores at both baseline and the 3-year follow-up, along with the corresponding change in score (baseline minus follow-up score). The NEI VFQ-25 composite and all subscales were unchanged between baseline and 3-year follow-up (except for ocular pain which increased slightly). Table 3
Table 1 Demographic and cognitive status characteristics of the sample, $N=365$

\begin{tabular}{ll}
\hline Characteristic & $n(\%)$ \\
\hline Age, years, $n$ (\%) & $246(67.4)$ \\
$60-69$ & $110(30.1)$ \\
$70-79$ & $9(2.5)$ \\
$80-89$ & \\
Gender, $n$ (\%) & $123(33.7)$ \\
Men & $242(66.3)$ \\
Women & \\
Race/ethnicity & $345(94.5)$ \\
White, non-Hispanic & $16(4.4)$ \\
African American & $4(1.1)$ \\
Other & \\
Cognitive status, MMSE score & $281(77.0)$ \\
$28-30$ & $57(15.6)$ \\
$26-27$ & $20(5.5)$ \\
$24-25$ & $7(1.9)$ \\
$\leq 23$ &
\end{tabular}

provides the analogous information for the LLQ. The LLQ composite and 5 of 6 LLQ subscales decreased between baseline and 3 years later (all $p<0.007$ ). The exception was the general dim lighting subscale $(p=$ 0.0579), which did not reach statistical significance.

Although the scores for the LLQ composite and 5 subscales decreased over 3 years, the decrease on average was small, approximately 1 to 3 points, leading one to question its clinical or practical significance. Thus we defined a clinically significant decrease in LLQ score to be a decrease of $\geq 5$ points from baseline to 3-year follow up. Our rationale was based on an accepted approach to determining minimally important changes in healthrelated quality of life, namely defining it as approximately half a standard deviation [26]. The standard deviation for the LLQ composite was approximately 10 , and thus we defined our minimally important difference as 5 points on the LLQ. Eighty-five of 365 (23.3\%) participants had $\geq 5$ point decrease in the LLQ composite between baseline and follow-up (Table 4), which is significantly larger than the 41 participants $(11.2 \%)$ who had $\geq 5$ point decrease on the NEI VFQ-25 composite over the same time period, $p<0.0001$. The seven individuals with MMSE scores $<24$ (indicating cognitive impairment) had greater decreases on both the NEI VFQ-25 and LLQ at the 3-year followup, as compared to those with MMSE scores $\geq 24$, although this association did not reach statistical significance for the LLQ $(p=0.0024$ and $p=0.0801$, respectively). The pattern of results in the tables did not change when these seven persons were dropped from the analysis. 
Table 2 NEI-VFQ Composite and Subscales scores at baseline and follow-up for participants in normal macular health group and the change in scores over this 3-year period $(N=365)$

\begin{tabular}{|c|c|c|c|c|}
\hline \multirow{3}{*}{ NEI-VFQ subscales } & \multicolumn{4}{|c|}{ Normal macular health at baseline ${ }^{a}$} \\
\hline & Baseline score & 3-year follow-up score & Difference $^{a}$ & $P$-value \\
\hline & \multicolumn{4}{|l|}{$\mathrm{M}(\mathrm{SD})$} \\
\hline Composite & $93.9(5.5)$ & $93.9(5.9)$ & 0.0 & 0.9875 \\
\hline General health & $73.9(21.7)$ & $72.8(21.7)$ & 1.1 & 0.2724 \\
\hline General vision & $83.7(11.2)$ & $82.5(11.7)$ & 1.2 & 0.0781 \\
\hline Near vision & $92.3(11.1)$ & $92.7(11.4)$ & -0.5 & 0.4984 \\
\hline Distance vision & $93.0(8.9)$ & $92.8(10.0)$ & 0.2 & 0.6675 \\
\hline Driving & $89.7(10.9)$ & $90.1(10.6)$ & -0.3 & 0.5635 \\
\hline Peripheral vision & $94.9(11.9)$ & $94.7(12.5)$ & 0.3 & 0.6773 \\
\hline Color vision & $97.9(8.85$ & $98.4(7.4)$ & -0.3 & 0.3699 \\
\hline Ocular Pain & $90.3(12.0)$ & $91.8(11.7)$ & -1.4 & 0.0221 \\
\hline \multicolumn{5}{|l|}{ Vision-specific } \\
\hline Role difficulties & $96.0(9.8)$ & 95.7 (10.6) & 0.3 & 0.6139 \\
\hline Dependency & $99.4(3.5)$ & $99.0(5.0)$ & 0.4 & 0.1419 \\
\hline Social functioning & $98.7(5.0)$ & $99.1(4.5)$ & -0.3 & 0.3565 \\
\hline Mental health & $94.5(8.1)$ & $94.0(10.5)$ & 0.5 & 0.3654 \\
\hline
\end{tabular}

${ }^{a}$ Difference is baseline score minus follow-up score

We examined whether visual function (visual acuity, contrast sensitivity, macular light sensitivity, low luminance deficit, rod-mediated dark adaptation) at baseline and change in these visual functions from baseline to follow-up were associated with participants who had $\geq 5$ point decrease in LLQ composite score at 3 years (Table 5). No aspect of visual function tested at baseline or its change score was related to those who had $\geq 5$ point LLQ composite drops. This was also the case for the NEI VFQ25 with one exception; a $0.10 \log$ MAR decline in visual acuity was associated with a $\geq 5$-point decrease on the NEI VFQ-25, as compared to those who declined $<5$ points.

\section{Discussion}

Our results suggest that a vision-targeted health-related quality of life questionnaire whose content is focused on night-time and low luminance visual activities is more likely to show a decline over time in older adults than a questionnaire that focuses on daytime activities. Older adults' composite scores were more likely to have a practically significant decrease over 3 years using a questionnaire that focused on low luminance activities (LLQ) than one focused on daytime activities (NEI VFQ-25). In addition, scores on five of six subscales of the LLQ significantly declined 3 years later, however none of the twelve subscales of NEI VFQ-25 declined over the same

Table 3 LLQ Composite and Subscales scores at baseline and follow-up for participants in normal macular health group and the change in scores over this 3-year period $(N=365)$

\begin{tabular}{|c|c|c|c|c|}
\hline \multirow{3}{*}{ LLQ subscales } & \multicolumn{4}{|c|}{ Normal macular health at baseline ${ }^{\text {a }}$} \\
\hline & Baseline score & 3-year follow-up score & Difference $^{a}$ & $P$-value \\
\hline & \multicolumn{4}{|l|}{$M(S D)$} \\
\hline Composite & $91.4(8.8)$ & $89.7(10.3)$ & 1.7 & $<0.0001$ \\
\hline Driving & $85.0(19.0)$ & $81.8(22.8)$ & 3.1 & 0.0009 \\
\hline Extreme lighting conditions & $87.2(11.2)$ & $85.0(13.5)$ & 2.2 & 0.0005 \\
\hline Mobility & $96.0(7.5)$ & $94.6(8.9)$ & 1.4 & 0.0014 \\
\hline Emotional distress & $97.6(6.2)$ & $96.3(7.9)$ & 1.2 & 0.0023 \\
\hline General dim-lighting & $91.5(11.4)$ & $90.5(11.8)$ & 1.0 & 0.0579 \\
\hline Peripheral vision & $93.4(12.3)$ & $91.4(14.8)$ & 2.0 & 0.0065 \\
\hline
\end{tabular}

\footnotetext{
${ }^{a}$ Difference is baseline score minus follow-up score
} 
Table 4 Percentage of participants having a $\geq 5$ point decrease in the NEI VFQ-25 composite as compared to the LLQ composite over 3 years

\begin{tabular}{llll}
\hline & $\begin{array}{l}\text { Composite decreased } \\
\text { by } \geq 5 \text { points } \\
n(\%)\end{array}$ & $\begin{array}{l}\text { Composite did not } \\
\text { decrease by } \geq 5 \text { points }\end{array}$ & $p$-value \\
\hline NEI VFQ-25 & $41(11.2)$ & $324(88.8)$ & $<0.0001$ \\
LLQ & $85(23.3)$ & $280(76.7)$ & \\
\hline
\end{tabular}

period. Our results imply that the use of questionnaires in clinical vision research on older adults that exclusively target daytime visual activities are likely to miss certain types and the extent of visibility problems older adults experience in daily life. It is noteworthy that this pattern of results emerged even in older adults in normal eye health, free of the common eye conditions of aging. Common ageing-related eye conditions such as AMD and glaucoma are associated with visual deficits under mesopic and scotopic conditions, which are more severe than what would be expected through aging alone $[14,27-29]$. It remains to be determined whether changes in the LLQ are associated with patients' perceptions about the worsening of low luminance vision in these conditions, as they progress.

This greater tendency for older adults' self-report activity problems under low luminance than in photopic conditions is consistent with psychophysical reports that older adults' visibility problems are accentuated under mesopic and scotopic conditions, as compared to younger adults $[7-9,30]$. However, in this study there was no association between psychophysically measured deficits and self-reported deficits as reported on the LLQ. Those persons who had worse visual function at baseline or had more visual decrease over 3 years, were not more likely to be those who had larger decreases on the LLQ over the 3 year period. This study focused on older adults in normal health, and it is possible that psychophysical testing is not sensitive enough to quantitate the low luminance difficulties in everyday life older adults in good eye health experience subjectively.

\section{Conclusions}

How patients view their own visual task difficulties and the emotional consequences these challenges present is an appropriate part of understanding the impact of eye conditions and aging on everyday life. Patient reported outcomes, such as questionnaires on vision-targeted health-related quality of life, are an essential part of evaluating the impact of ophthalmic interventions to slow the progression of age-related eye conditions such as AMD, glaucoma, and diabetic retinopathy. Yet also part of understanding the role of patient reported outcomes is the transition of aging-associated conditions from normal eye health to their earliest incident emergence. Thus, it is important to recognize, that even in

Table 5 Vision variables stratified by participants with a $\geq 5$ point decrease in the LLQ composite from baseline to 3-year follow-up versus those whose scores did not decrease by $\geq 5$ points

\begin{tabular}{|c|c|c|c|c|c|c|}
\hline & $\begin{array}{l}\text { LLQ composite decreased } \\
\text { by } \geq 5 \text { points } \\
N=85\end{array}$ & $\begin{array}{l}\text { LLQ composite did not } \\
\text { decrease by } \geq 5 \text { points } \\
N=280\end{array}$ & $p$-value & $\begin{array}{l}\text { NEI VFQ- } 25 \text { composite } \\
\text { decreased by } \geq 5 \text { points } \\
N=\end{array}$ & $\begin{array}{l}\text { NEI VFQ- } 25 \text { composite did } \\
\text { not decrease by } \geq 5 \text { points } \\
N=\end{array}$ & p-value \\
\hline & \multicolumn{6}{|c|}{ Mean (standard deviation) unless otherwise noted } \\
\hline $\begin{array}{l}\text { Visual acuity at baseline } \\
\text { (logMAR) }\end{array}$ & -0.02 & -0.02 & 0.9908 & 0.00 & -0.02 & 0.1196 \\
\hline Change in visual acuty ${ }^{a}$ & -0.06 & -0.04 & 0.4341 & -0.10 & -0.04 & 0.0145 \\
\hline $\begin{array}{l}\text { Contrast sensitivity, log } \\
\text { sensitivity }\end{array}$ & 1.63 & 1.65 & 0.1482 & 1.65 & 1.64 & 0.7051 \\
\hline $\begin{array}{l}\text { Change in contrast } \\
\text { sensitivity }\end{array}$ & 0.05 & 0.04 & 0.7315 & 0.06 & 0.04 & 0.3192 \\
\hline Light sensitivity, dB b & 30.4 & 30.6 & 0.3264 & 30.5 & 30.6 & 0.8041 \\
\hline Low luminance deficit & 0.30 & 0.29 & 0.5884 & 0.31 & 0.29 & 0.2780 \\
\hline $\begin{array}{l}\text { Change in low } \\
\text { luminance deficit }\end{array}$ & -0.05 & -0.04 & 0.7105 & -0.08 & -0.04 & 0.1039 \\
\hline $\begin{array}{l}\text { Rod-mediated dark } \\
\text { adaptation, rod intercept } \\
\text { (minutes) }\end{array}$ & 10.45 & 10.10 & 0.4050 & 10.1 & 10.2 & 0.8394 \\
\hline $\begin{array}{l}\text { Change in rod-mediated } \\
\text { dark adaptation }\end{array}$ & -1.26 & -0.85 & 0.3121 & -1.8 & -0.8 & 0.0832 \\
\hline
\end{tabular}

${ }^{a}$ Change in visual function variables defined as the value at baseline minus the value at the 3-year follow-up

${ }^{b}$ Light sensitivity was only measured at baseline 
aging, low luminance activities already play a significant role in reducing vision-targeted health related quality of life, and a health-related quality of life questionnaire reflects this trend.

\begin{abstract}
Abbreviations
AMD, age-related macular degeneration; ALSTAR, Alabama Study on Early Age-Related Macular Degeneration; AREDS, Age-Related Eye Disease Study; $\mathrm{dB}$, decibels; LLQ, Low Luminance Questionnaire; logMAR, logarithm of the minimum angle of resolution; MMSE " Mini-mental State Examination; NEI VFQ-25, National Eye Institute Visual Functioning Questionnaire (25-item version); $\cup A B$, University of Alabama at Birmingham
\end{abstract}

\section{Acknowledgements}

None.

\section{Funding}

This research was supported by grants from the National Institute on Aging (R01AG04212), Research to Prevent Blindness, and the EyeSight Foundation of Alabama.

\section{Availability of data and materials}

The data have not yet been placed in a publicly available repository since the investigators are still in the process of analyzing and publishing the ALSTAR study findings.

\section{Authors' contributions}

CO designed the study, oversaw data collection, interpreted the data, and drafted the manuscript. GMGJ designed the study, designed the statistical analyses of the data, interpreted the data, and drafted the manuscript. Both authors read and approved the final manuscript.

\section{Competing interests}

The authors declare that they have no competing interests.

\section{Consent for publication}

No personal data or clinical data associated with personal data are presented in this manuscript.

\section{Ethics approval and consent to participate}

The protocol (\#F080205001) was approved by the Institutional Review Board of the University of Alabama at Birmingham (UAB) and followed the tenants of the Declaration of Helsinki. Informed consent was obtained from all participants after the nature and purpose of the study was described.

\section{Author details}

'Department of Ophthalmology, School of Medicine, University of Alabama at Birmingham, Birmingham, AL 35294-0009, USA. ${ }^{2}$ Department of Epidemiology, School of Public Health, University of Alabama at Birmingham, Birmingham, AL 35294-0022, USA.

Received: 26 January 2016 Accepted: 23 June 2016

Published online: 07 July 2016

\section{References}

1. Ball K, Owsley C, Stalvey B, Roenker DL, Sloane M, Graves M. Driving avoidance and functional impairment in older drivers. Accid Anal Prev. 1998:30:313-22.

2. Mangione CM, Lee PP, Pitts J, Gutierrez P, Berry S, Hays RD, National Eye Institute Visual Function Questionnaire Field Test Investigators. Psychometric properties of the National Eye Institute visual function questionnaire (NEI-VFQ). Arch Ophthalmol. 1998;116:1496-504.

3. Betz ME, Carpenter CR, Genco E, Carr DB. Driving self restriction and age: a study of emergency department patients. Inj Epidemiol. 2014;1:18.

4. Kosnik W, Winslow L, Kline D, Rasinski K, Sekuler R. Visual changes in daily life throughout adulthood. J Gerontol B Psychol Sci Soc Sci. 1988;43:P63-70.

5. Jackson GR, Owsley C, Cordle EP, Finley CD. Aging and scotopic sensitivity. Vision Res. 1998;38:3655-62.

6. Jackson GR, Owsley C, McGwin GJ. Aging and dark adaptation. Vision Res. 1999:39:3975-82.
7. Sloane ME, Owsley C, Alvarez SL. Aging, senile miosis and spatial contrast sensitivity at low luminance. Vision Res. 1988;28:1235-46.

8. Sturr JF, Kline GE, Taub HA. Performance of young and older drivers on a static acuity test under photopic and mesopic luminance conditions. Hum Factors. 1990:32:1-8

9. Haegerstrom-Portnoy G, Schneck ME, Brabyn JA. Seeing into old age: vision function beyond acuity. Optom Vis Sci. 1999;76:141-58.

10. Richards OW. Effects of luminance and contrast on visual acuity, ages 16 to 90 years. Am J Optom Physiol Opt. 1977:54:178-84.

11. Mangione CM, Lee PP, Gutierrez PR, Spritzer K, Berry S, Hays RD, NEI VFQ Field Test Investigators. Development of the 25-item National Eye Institute Visual Function Questionnaire. Arch Ophthalmol. 2001;119:1050-8.

12. Mangione CM, Phillips RS, Seddon JM, Lawrence MG, Cook EF, Dailey R, Goldman L. Development of the activities of daily vision scale: A measure of visual functional status. Med Care. 1992;30:1111-26.

13. Steinberg EP, Tielsch JM, Schein OD, Javitt JC, Sharkey P, Cassard SD, Legro MW, Diener-West M, Bass EB, Damiano AM, et al. The VF-14: an index of functional impairment in patients with cataract. Arch Ophthalmol. 1994; 112:630-8.

14. Owsley C, McGwin Jr G, Scilley K, Kallies K. Development of a questionnaire to assess vision problems under low luminance in age-related maculopathy. Invest Ophthalmol Vis Sci. 2006:47:528-35.

15. Finger RP, Fenwick E, Owsley C, Holz FG, Lamoureux EL. Visual functioning and quality of life under low luminance: evaluation of the German Low Luminance Questionnaire. Invest Ophthalmol Vis Sci. 2011;52:8241-9.

16. Owsley C, Huisingh C, Jackson GR, Curcio CA, Szalai AJ, Dashti N, Clark M, Rookard K, McCrory MA, Wright TT, et al. Associations between abnormal rod-mediated dark adaptation and health and functioning in older adults with normal macular health. Invest Ophthalmol Vis Sci. 2014;55:4776-89.

17. Owsley C, McGwin Jr G, Clark ME, Jackson GR, Callahan MA, Kline LB, Witherspoon CD, Curcio CA. Delayed rod-mediated dark adaptation is a functional biomarker for incident early age-related macular degeneration. Ophthalmology. 2016:123:344-51.

18. Age-Related Eye Disease Study Research Group. The Age-Related Eye Disease Study severity scale for age-related macular degeneration. AREDS Report No. 17. Arch Ophthalmol. 2005;123:1484-98.

19. Cappelleri JC, Lundy JJ, Hays RD. Overview of classical test theory and item response theory for the quantitative assessment of items in developing patient-reported outcomes measures. Clin Ther. 2014;36:648-62.

20. Folstein MF, Folstein SW, McHugh PR. "Mini-mental state": a practical method for grading the cognitive state of patients for the clinician. J Psychiatr Res. 1975;12:189-98.

21. Beck RW, Moke PS, Turpin AH, Ferris FLI, SanGiovanni JP, Johnson CA, Chandler DL, Cox TA, Blair RC, Kraker RT. A computerized method of visual acuity testing: adaptation of the early treatment of diabetic retinopathy study testing protocol. Am J Ophthalmol. 2003;135:194-205.

22. Sunness JS, Rubin GS, Applegate CA, Bressler NM, Marsh MJ, Hawkins BS, Haselwood D. Visual function abnormalities and prognosis in eyes with age-related geographic atrophy of the macula and good visual acuity. Ophthalmology. 1997;104:1677-91.

23. Pelli DG, Robson JG, Wilkins AJ. The design of a new letter chart for measuring contrast sensitivity. Clin Vis Sci. 1988;2:187-99.

24. Elliott DB, Bullimore MA, Bailey IL. Improving the reliability of the Pelli-Robson contrast sensitivity test. Clin Vis Sci. 1991;6:471-5.

25. Owsley C, Huisingh C, Clark ME, Jackson GR, McGwin GJ. Comparison of visual function in older eyes in the earliest stages of age-related macular degeneration to those in normal macular health. Curr Eye Res $h$. 2016;41:266-72

26. Norman GR, Sloan JA, Wyrwich KW. Interpretation of changes in health-related quality of life: the remarkable universality of half a standard deviation. Med Care. 2003:41:582-92.

27. Drum B, Armaly MF, Huppert W. Scotopic sensitivity loss in glaucoma. Arch Ophthalmol. 1986:104:712-7.

28. Glovinsky Y, Quigley HA, Drum B, Bissett RA, Jampel HD. A whole-field scotopic retinal sensitivity test for the detection of early glaucoma damage. Arch Ophthalmol. 1992:110:486-90.

29. Wu Z, Guymer RH, Finger RP. Low luminance deficit and night vision symptoms in intermediate age-related macular degeneraiton. $\mathrm{Br}$ Ophthalmol. 2015;100:395-8

30. Jackson GR, Owsley C. Scotopic sensitivity during adulthood. Vision Res. 2000;40:2467-73. 